\title{
Aerobic and anaerobic training effects on the antioxidant enzymes of the blood
}

\author{
Semih Selamoglu${ }^{1}$, Faruk Turgay ${ }^{1}$, Berkant Muammer Kayatekin ${ }^{2}$, \\ Sevil Gönenc ${ }^{2}$, Çetin Yslegen ${ }^{3}$ \\ ${ }^{1}$ Izmir Sports Health Center \\ ${ }^{2}$ Department of Physiology, Dokuz Eylül University Medical School \\ ${ }^{3}$ Department of Sports Medicine, Ege University Medical School, Izmir, Turkey
}

Received: September 4, 2000

Accepted: December 18, 2000

\begin{abstract}
The purpose of the present study was to investigate the effects of aerobic and anaerobic training on serum lipid peroxidation levels and on antioxidant enzyme activities.

Long distance runners for aerobic training group, and wrestlers for anaerobic training group were chosen. Non-sporting men were used as control group. When the aerobic power was compared; indirect $\mathrm{VO}_{2} \max$ of long-distance runners were found higher than wrestlers and control group $(\mathrm{p}<0.001, \mathrm{p}<0.001)$. When lipid peroxidation levels were compared; levels of the thiobarbituric acid reactive substances (TBARS) of long distance runners were found to be lower than those in the control group $(\mathrm{p}<0.05)$, but similar to those found in wrestlers. Comparison of antioxidant enzyme activities in erythrocytes show that there were no significant difference among the groups in superoxide dismutase enzyme activities, but glutathione peroxidase (GPx) activity of long distance runners was higher than that measured in wrestlers $(\mathrm{p}<0.05)$.

These results suggest that aerobic training increased in erythrocytes GPx activity with a subsequent decrease in plasma TBARS levels but anaerobic training had no effect on this process.

Keywords: superoxide dismutase, glutathione peroxidase, thiobarbituric acid, reactive substances, exercise, aerobic and anaerobic training, non sporting men, long-distance runners, antioxidant enzyme activities
\end{abstract}

Increased energy demand during physical exercise, especially of the aerobic type, necessitates a multifold increase in oxygen supply to active tissues. During exercise,

Correspondence should be addressed to

Berkant Muammer Kayatekin, MD

Department of Physiology,

Dokuz Eylül University Medical School

35340 Balçova, Izmir, Turkey

Fax: 00-90-232-2590541

E-mail: kayabm@deu.edu.tr 
bodily oxygen consumption is greatly increased, up to 10 - to 15 -fold greater than resting levels [9]. Oxygen-centred radicals are produced in the intermediate metabolism [4]. Most of the oxygen consumed in the mitochondria is utilized to produce adenosine 5'triphosphate, but during oxidative phosphorylation the superoxide radicals, hydrogen peroxide, and hydroxyl radicals are produced by the univalent reduction of oxygen and leak out of the electron transfer chain [6]. As a whole, they are classified as reactive oxygen species (ROS) and are responsible for a series of biochemical and physiological changes, namely oxidative stress. The ROS released cause the lipid peroxidation of polyunsaturated fatty acids in the biological membranes and blood, inducing alterations of the cell functions [7]. Lipid peroxides readily decompose to liberate highly reactive carbonyle fragments such as malondialdehyde. Malondialdehyde (MDA) was the major species responsible for thiobarbituric acid reactive substances (TBARS) [8].

Strenuous physical exercise induces oxidative damage to lipids in various tissues $[22,23]$. In resting state the body is equipped with both non-enzymatic and enzymatic antioxidant reserves to prevent the potentially harmful effects of ROS [13]. The fine physiological balance between oxidative reactions and antioxidant capacity may be perturbed by intense physical activity. Antioxidant defence systems preserve homeostasis for normal cell function at rest and perhaps during mild-oxidative stress. Primary components of the physiological antioxidant defence are superoxide dismutase (SOD), catalase and glutathione peroxidase (GPx). SOD catalyzes the dismutation of superoxide to $\mathrm{O}_{2}$ and $\mathrm{H}_{2} \mathrm{O}_{2}$, which catalase (CAT) converts to water and $\mathrm{O}_{2}$. GPx can reduce $\mathrm{H}_{2} \mathrm{O}_{2}$ to form glutathione disulphide and water [5].

Large number of studies have tested the effect of a variety of endurance exercise training regimens on antioxidant defences, but information on the effect of anaerobic training on antioxidant defences is scanty. The aim of the present study was to assess the effects of aerobic and anaerobic training on serum lipid peroxidation levels and on antioxidant enzymatic activities in erythrocytes.

\section{Materials and Methods}

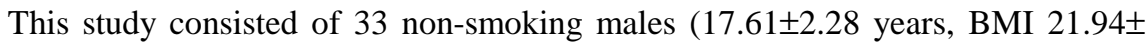
0.63 ); including 11 long-distance runners (aerobic training group) and 11 wrestlers (anaerobic training group) who have been doing sport for average $5.18 \pm 0.70$ years on the awerage and in control group. The control subjects did not perform any regular physical activity before the study. All the experiments were performed in according to the Helsinki Declaration.

Subjects attended the laboratory in the morning, after a $12 \mathrm{~h}$ fast and a $10 \mathrm{ml}$ blood sample was obtained from an antecubital vein. Blood samples were collected 48 
hours after the termination of the training in order to minimise the residual effect of the last exercise. Heparinized venous whole blood was used for measuring erythrocyte antioxidant enzymes (SOD and GPx) activities. Blood hemoglobin values for calculating enzyme activities were determined by using Coulter Counter. Plasma TBARS levels were measured as an indicator of lipid peroxidation.

\section{Determination of SOD activity}

Erythrocyte SOD was determined with a Randox test combination (Randox, Crumlin, UK). Xanthine and xanthine oxidase were used to generate superoxide radicals reacting with 2-(4-iodophenyl) 3-(4-nitrophenol)-5 phenyl tetrazolium chloride (INT) to form a red formazan dye. The concentration of the substrates were $0.075 \mu \mathrm{mol}$ for xanthine and $0.037 \mathrm{mmol}$ for INT. Superoxide dismutase inhibits this reaction by converting the superoxide radical to oxygen. A SOD unit inhibits the rate of reduction of INT by 50\% in a complex system with xanthine and xanthine oxidase. Because of the small linearity range of the test, the sample was diluted so that the percentage of inhibition fell between $30 \%$ and $60 \%$. A standard curve was prepared, using the kit standard, and the value for the diluted sample was read from this curve. SOD activity was measured at $505 \mathrm{~nm}$ on a Shimadzu UV-1201v spectrometer on hemolysates of washed erythrocytes obtained by centrifugation of whole blood. Results were expressed in SOD U/g hemoglobin.

\section{Determination of GPx activity}

GPx was also determined with a Randox test combination (Randox, Crumlin, UK). GPx catalyses the oxidation of glutathione (at a concentration of $5 \mathrm{mmol}$ ) by cumene hydroperoxide according to the method of Paglia and Valentine [21]. In the presence of glutathione reductase (at a concentration $>0.75 \times 10-3 \mathrm{U}$ ) and $0.35 \mathrm{mmol}$ NADPH, the oxidised glutathione was immediately converted to the reduced form with a concomitant oxidation of NADPH to $\mathrm{NADP}^{+}$. The decrease in absorbance at $340 \mathrm{~nm}$ was measured at $37^{\circ} \mathrm{C}$. The assay was performed on a hemolysate of washed erythrocytes obtained from the mixing of $0.05 \mathrm{ml}$ whole blood with $1 \mathrm{ml}$ cold diluting agent and $1 \mathrm{ml}$ Drabkin reagent. The GPx unit was defined as the enzyme activity necessary to convert $1 \mathrm{mmol}$ of NADPH to NADP in 1 minute. The activity of GPx is expressed in $\mathrm{U} / \mathrm{g}$ hemoglobin. 
Determination of TBARS level

In a modified Yagi method [24], $0.05 \mathrm{ml}$ of blood was sampled with a pipette for determination of blood cells and placed in $1.0 \mathrm{ml}$ of normal saline in a centrifuge tube. After gently shaking, the tube was spun at $3000 \mathrm{rpm}$ for 10 minutes and $0.5 \mathrm{ml}$ of the supernatant was transferred to another centrifuge tube. The $4.0 \mathrm{ml}$ of $1 / 12 \mathrm{~N} \mathrm{H}_{2} \mathrm{SO}_{4}$ was added to this solution and the mixture was shaken gently. Then $0.5 \mathrm{ml}$ of $10 \%$ phosphotungstic acid was added and mixed. After standing at room temperature for 5 minutes, the mixture was centrifuged at $3000 \mathrm{rpm}$ for $10 \mathrm{~min}$. After the supernatant was discarded, the sediment was mixed with $2.0 \mathrm{ml}$ of $\mathrm{N} / 12 \mathrm{H}_{2} \mathrm{SO}_{4}$ and $0.3 \mathrm{ml}$ of $10 \%$ phosphotungstic acid and the mixture was centrifuged. The sediment was suspended in $4.0 \mathrm{ml}$ of distilled water and $1.0 \mathrm{ml}$ of thiobarbituric acid (TBA) reagent was added. The reaction mixture was heated for $60 \mathrm{~min}$ at $100{ }^{\circ} \mathrm{C}$ in a water bath. After cooling with tap water, $5.0 \mathrm{ml}$ of $\mathrm{n}$-butanol was added and the mixture was shaken vigorously, then centrifuged at $3000 \mathrm{rpm}$ for $15 \mathrm{~min}$. Finally, the n-butanol layer was taken for spectophotometric measurement at $532 \mathrm{~nm}$. A standard curve was prepared using the MDA standard (1, 1, 3, 3-tetraethoxypropane) and the value for the plasma was read from this curve. The results were expressed as nmol/ml.

Determination of $\mathrm{VO}_{2} \max$

$\mathrm{VO}_{2}$ max was determined indirectly by Astrand's method using Monarck bicycle ergometer [3].

\section{Statistical analysis}

All results were expressed as mean \pm S.E.M. The statistical analysis of the data was performed using Mann-Whitney U test. The significance was set at $\mathrm{p}<0.05$.

\section{Results}

When the effect of exercise on aerobic power was examined; indirect $\mathrm{VO}_{2}$ max of long-distance runners were found higher than wrestlers and non-sporting men (respectively, $\mathrm{p}<0.001, \mathrm{p}<0.001$, Table I). 
Table I

Indirect $\mathrm{VO}_{2}$ max values, SOD, GPx activities and TBARS levels of sportsmen and of the control group

\begin{tabular}{lllll}
\hline Groups & $\begin{array}{c}\mathrm{VO}_{2} \max \\
(\mathrm{ml} / \mathrm{kg} / \mathrm{min})\end{array}$ & \multicolumn{1}{c}{$\begin{array}{c}\mathrm{SOD} \\
(\mathrm{U} / \mathrm{gHb})\end{array}$} & $\begin{array}{c}\text { GPx } \\
(\mathrm{U} / \mathrm{gHb})\end{array}$ & $\begin{array}{c}\text { TBARS } \\
(\mathrm{nmol} / \mathrm{ml})\end{array}$ \\
\hline Control & $47.40 \pm 1.57$ & $1150.36 \pm 51.60$ & $42.96 \pm 4.86$ & $1.43 \pm 0.09$ \\
Distance runners & $62.30 \pm 1.85^{* * *}$ & $1059.27 \pm 111.29$ & $56.62 \pm 3.68^{* *}$ & $1.08 \pm 0.10^{*}$ \\
Wrestlers & $49.71 \pm 1.49$ & $1269.61 \pm 66.44$ & $45.54 \pm 3.13$ & $1.15 \pm 0.15$ \\
\hline
\end{tabular}

Results were presented as means \pm S.E.M.

* Lower than control $\mathrm{p}<0.05$

$* *$ Higher than wrestlers $\mathrm{p}<0.05$

$* * *$ Higher than wrestlers and control $\mathrm{p}<0.001$

When plasma lipid peroxidation levels and antioxidant enzymatic activities were investigated; TBARS levels of long-distance runners were lower than those of nonsporting men $(\mathrm{p}<0.05)$ but similar to those measured in wrestlers. GPx enzyme activity of long-distance runners was higher than those of wrestlers $(\mathrm{p}<0.05)$ but similar to those found in the non-sporting males. There were no significant difference among the groups in SOD enzyme activities (Table I).

A positive correlation between the subject's $\mathrm{VO}_{2} \max$ and GPx activity was found $(\mathrm{r}=0.53, \mathrm{p}<0.01)$. A negative correlation between the subject's $\mathrm{VO}_{2} \mathrm{max}$ and plasma MDA level was found $(r=-0.38, p<0.05)$.

\section{Discussion}

Increased oxygen utilization during exercise cause generation of free radicals [11]. Chronic aerobic training has been claimed to reduce exercise-increased lipoperoxidation by improving the body's defence capabilities against free radicals generation, likely as a result of an adaptive increase in the activities of the scavenger enzyme systems [2]. Scientific literature on the effect of anaerobic training on antioxidant defence system and on lipid peroxidation is scanty [17].

Human erythrocytes are well equipped with the enzymes SOD, catalase and GPx, that protect the cells against the accumulation of superoxide radical and/or hydrogen peroxide normally produced during the oxidation of hemoglobin [19]. It has been reported that endurance training elevates the antioxidant enzyme activities in blood at rest and during post-exercise recovery $[14,17]$. However, a controversy still exists as to which enzyme and under what condition an enzyme can be activated [11, 12, 15, 16]. Available data suggest that each of the antioxidant systems may have a different 
response to acute and chronic exercise depending upon their biochemical and molecular mechanism of regulation [10]. Mena et al. described that under resting conditions the SOD and GPx activities were higher in cyclists than in the control group [18]. Marzatico et al. determined that blood SOD and GPx activities were higher in marathon runners and sprinters than in controls [17]. Ohno et al. have reported that in sedentary students after a brief physical exercise no increase in erythrocyte SOD activity was found [19]. In another study, resting blood SOD and GPx activities were no different in jump-trained (volleyball players) compared with untrained subjects [20]. It has been reported that blood GPx activity increased after swim training program in mice [14].

The results from the present investigation demonstrated that SOD activity was unaffected by aerobic and anaerobic training. GPx enzyme activities of long-distance runners were higher than those detected in wrestlers. Despite exercise-induced free radical changes, there is a positive side to oxidative stress associated with regular exercise [1]. Subjects with high aerobic power show significantly greater antioxidant enzyme activity $[17,23]$. It has been reported that there exist a good correlation between exercise endurance time and GPx activity [1]. In this study, a positive correlation between the subject's $\mathrm{VO}_{2} \max$ and GPx activity was found. This could further show how even aerobic training is able to prevent the toxic effects of lipid peroxidation.

MDA is the end product of lipid peroxidation and is a well-known parameter for determining the increased free radical formation in the body. It has been reported that lipid peroxidation levels are lower in endurance-trained than in untrained animals [2]. The knowledge on the effect of training on lipid peroxidation in humans is sparse equivocal. Ohno et al. [19] and Jenkins et al. [12] determined that plasma MDA levels were decreased related to training adaptation. However, Marzatico et al. found that resting plasma MDA levels of marathon runners and sprinters are higher than control levels [17]. Results of studies on lipid peroxidation induced by exercise are actually inconsistent due to the wide variety of methods employed and the differences in exercise protocols (e.g. type, duration and intensity of exercise). It was observed that resting plasma MDA negative by correlated with the aerobic capacity of the individuals, suggesting a protective effect of physical fitness [23].

In the present study, when resting plasma lipid peroxidation levels were examined; a negative correlation between the subject's $\mathrm{VO}_{2} \max$ and plasma TBARS level was found. TBARS levels of long distance runners were lower than control levels but TBARS levels of wrestlers did not differ from those found in controls.

In conclusion, these results suggest that aerobic training increased GPx activity in erythrocytes with a subsequent decrease in plasma TBARS levels while anaerobic training had no effect on this process. 


\section{REFERENCES}

1. Alessio, H.M.: Exercise-induced oxidative stress. Med. Sci. Sports Exerc., 25, 218-224 (1993).

2. Alessio, H., Goldfarb, A.: Lipid peroxidation and scavenger enzymes during exercise: adaptive response to training. J. Appl. Physiol., 64, 1333-1336 (1988).

3. Åstrand, P.O., Rodalhl, K.: Textbook of Work Physiology. New York, McGraw Hill, 1986.

4. Bast, A., Haenen, G.R.M.M., Doelman, C.S.A.: Oxidant and antioxidants. Am. J. Med., 91, 2-13 (1994).

5. Benzi, G.: Aerobic performance and oxygen free-radicals. J. Sports Med. and Physical Fitness, 33, 205-222 (1993).

6. Change, B., Sies, H., Boveris, A.: Hydroperoxide metabolism in mammalian organs. Physiol. Rev., 59, 527-605 (1979).

7. Duthie, G.G., Robertson, J.D., Maughaman, R.J., Morrice, C.P.: Blood antioxidant status and erythrocyte lipid peroxidation following distance running. Arch. Biochem. Biophys., 282, 78-83 (1990).

8. Gutteridge, J.M.C.: Free-radical induced damage to lipids, amino acids, carbohydrates and nucleic acids determined by thiobarbituric acid reactivity. Int. J. Biochem., 14, 649-653 (1982).

9. Halliwell, B., Gutteridge, J.M.C.: Free Radicals in Biology and Medicine. 3rd ed. New York, Oxford University Press Inc., 1999.

10. Harris, E.D.: Regulation of antioxidant enzymes. FASEB J., 6, 2675-2683 (1992).

11. Jenkins, R.R.: Free radical chemistry: Relation to exercise. Sports Medicine, 5, 156-170 (1988).

12. Jenkins, R.R., Friedland, R., Howald, H.: The relationship of oxygen consumption to superoxide dismutase and catalase activity in human skelatal muscle. Int. J. Sports Med., 4, 11-14 (1984).

13. Ji, L.L.: Oxidative stress during exercise: implications of antioxidant nutrients. Free Rad. Biol. Med., 18, 1079-1086 (1994).

14. Kanter, M.M., Hamlin, R.L., Unverferth, D.V., Davis, H.W., Merola, A.J.: Effect of exercise training on antioxidant enzymes and cardiotoxicity of doxorubicin. J. Appl. Physiol., 59, 1298-1303 (1985).

15. Laughlin, M.H., Simpson, T., Sexton, W.L., Brown, O.R., Smith, K., Korthuis, J.: Skelatal muscle oxidative capacity, antioxidant enzymes, and exercise training. J. Appl. Physiol., 68, 2337-2343 (1990).

16. Li, L.J.: Antioxidant enzyme response to exercise and aging. Med. Sci. Sports Exerc., 25, 225-231 (1993).

17. Marzatico F., Pansara, O., Bertorelli, L., Somenzini, L., Della Valle, G.: Blood free radical antioxidant enzymes and lipid peroxides following long-distance and lactacidemic performances in highly trained aerobic and sprint athletes. J. Sports Med. Phys. Fitness, 37, 235-239 (1997).

18. Mena, P., Maynar, M., Gutierrez, J.M., Maynar, J., Timon, J., Campillo, J.E.: Erythrocyte free radical scavenger enzymes in bicycle professional racers, adaptation to training. Int. J. Sports Med., 12, 563-566 (1991).

19. Ohno, H.Y., Sato, Y., Yamashita, K.: The effect of brief physical exercise on free radical scavenging enzyme systems in human red blood cells. Can. J. Physiol. Pharmacol., 64, 1263-1265 (1985).

20. Ørtenblad, N., Madsen, K., Djurhuus, M.S.: Antioxidant status and lipid peroxidation after short-term maximal exercise in trained and untrained humans. Am. J. Physiol., R1258-R1263 (1997).

21. Paglia, D.E., Valentine, W.N.: Studies on the quantitave and qualitative characterization of erythrocyte glutathione peroxidase. J. Lab. Clin. Med., 70, 158-169 (1967).

22. Radak, Z., Asano, K., Inoue, M., Kizaki, T., Oh-Ishi, S., Suzuki, K., Taniguchi, N., Ohno, H.: Superoxide dismutase derivative reduces oxidative damage in skeletal muscle of rats during exhaustive exercise. J. Appl. Physiol., 79, 129-135 (1995).

23. Sen, C.K.: Oxidants and antioxidants in exercise. J. Appl. Physiol., 79, 675-686 (1995).

24. Yagi, K.: Assay for blood plasma or serum. Methods in Enzymology, 105, 328-331 (1984). 